\title{
High Nanopore Volume Tetrethoxysilane Based Aerogels Prepared with Addition of N, N-Dimethylformamide at Different Stage of the Sol-Gel Process
}

\author{
Chaoshuai LEI *, Enshuang ZHANG, Hongyan HUANG, Xuyang JI, Lijuan HE, \\ Wenjing LI, Jieying YANG, Yingmin ZHAO, Hao ZHANG
}

Aerospace Institute of Advanced Materials \& Processing Technology, Beijing, 100071, China

crossref http://dx.doi.org/10.5755/j02.ms.24912

Received 16 December 2019; accepted 10 February 2020

\begin{abstract}
Using tetraethoxysilane (TEOS) as a precursor, silica aerogels were synthesized via the sol-gel polymerization followed by supercritical drying process. During the polymerization period, N, N-dimethylformamide (DMF), acting as a chemical additive for the structure control, was introduced in the hydrolysis step and condensation step, respectively. As a result, the nanopore volumes for the pores smaller than $100 \mathrm{~nm}$ were up to $6.0 \mathrm{~cm}^{3} / \mathrm{g}$ and $5.7 \mathrm{~cm}^{3} / \mathrm{g}$ for the samples that produced with DMF addition in the hydrolysis step and condensation step, while the value for the sample without DMF was only $4.6 \mathrm{~cm}^{3} / \mathrm{g}$. Besides, the sample with DMF addition in the condensation step possessed more uniform pore size distribution while compared with that with DMF addition in the hydrolysis step. DMF can provide a shielding layer around the colloid particles through hydrogen bonds, inhibiting the aggregation of colloid particles and the enlarging of pore sizes.
\end{abstract}

Keywords: silica aerogels, sol-gel process, supercritical drying, nanoporous structure, DMF.

\section{INTRODUCTION}

Silica aerogels are nano-porous materials that have gained extensive attention in the past decades, owing to their extraordinary properties, such as low density [1], large surface area [2], high porosity, and extremely low thermal conductivity [3,4]. For these extraordinary characteristics, silica aerogels are presently of interest in thermal insulation $[5,6]$, acoustic insulation $[7,8]$, catalysts and sensors [9-11]. These characteristics are derived from their mesoporous structures, which are formed in the sol-gel polymerization of silicon alkoxides, and preserved under supercritical drying or ambient pressure drying [12-14]. However, due to the aggregation of the colloidal particles, non-uniform pore structure often appears in the aerogel, accompanied by the existence of many large pores in the range of a few hundred nanometers which cannot be measured by $\mathrm{N}_{2}$ adsorption-desorption method generally $[15,16]$. Therefore, the nanopores volume in these silica aerogels with particle aggregation is restricted severely.

One of the facile ways to prepare the silica aerogels with uniform pore size is the introduction of drying control chemical additives (DCCAs) such as DMF [17-19]. He et al. synthesized the water-glass-based silica aerogel with uniform pore structure, and its pore volume was as high as $3.3 \mathrm{~cm}^{3} / \mathrm{g}$ after the addition of dimethylformamide (DMF) [18]. Similarly, Cai et al. prepared methyltrimethoxysilane based silica aerogels that exhibit uniform pore structures [20]. Using tetraethoxysilane (TEOS) as a precursor, Vollet et al. researched the structure of aerogels prepared by sonohydrolysis method with additions of DMF [21]. However, attributed to the ambient pressure drying

\footnotetext{
* Corresponding author. Tel.: +86-18310761101; fax: +01088534479.

E-mail address: leichaoshuai@126.com (C. Lei)
}

method, the pore volume of the aerogel in this study is very low [22]. In fact, the researchers found that the strong polar reagent seems work as not only the control additives but also a catalyst, modifying the hydrolysis and polymerization rates and ultimately affecting the structural properties [21]. DCCAs can decrease the hydrolysis rate by combining with water molecules [18], and increase the polymerization rate via the deprotonation. Furthermore, it can be absorbed on the silica clusters through the hydrogen bond interactions and provide the steric hindrance around the colloid particles [20]. This shielding effect can inhibit the aggregation of the silica sol particles, which will contribute to uniform pore structure of the silica aerogel. Whereas, the study about the modification on the nanopore volume of silica aerogel is limited. High nanopore volume is critical for high performance silica aerogels.

In this paper, with the addition of DMF as the structure control reagent, the monolithic silica aerogels with high nanopore volume and uniform pore structure were synthesized by the sol-gel polymerization of TEOS. In this aerogel, the content of large pores larger than $100 \mathrm{~nm}$ decrease remarkably. Besides, when DMF is added in the condensation step instead of the hydrolysis step, the pore structure in the aerogel is more uniform.

\section{EXPERIMENTAL}

Preparation of silica aerogels: Fig. 1 a shows the flow chart of the fabrication routes. The wet gels were prepared using the conventional two-step method. Firstly, the TEOS was hydrolysed in a mixed solution of $\mathrm{H}_{2} \mathrm{O}$, ethanol and $\mathrm{HCl}$ (molar ratio $\mathrm{n}_{\mathrm{TEOS}}: \mathrm{n}_{\mathrm{H} 2 \mathrm{O}}: \mathrm{n}_{\mathrm{ethanol}}: \mathrm{n}_{\mathrm{HCl}}=1: 4: 6: 8 \times 10^{-4}$ ) under refluxing at $50^{\circ} \mathrm{C}$ for $30 \mathrm{~min}$. Secondly, for the condensation step, the above-mentioned compounds met the overall molar ratio (TEOS: $\mathrm{H}_{2} \mathrm{O}$ : Ethanol: $\mathrm{NH}_{3}=1: 6: 8: 1 \times 10^{-2}$ ) with additional solution of $\mathrm{H}_{2} \mathrm{O}$, 
ethanol and ammonia hydroxide. The silica aerogels were obtained by the supercritical drying method after aging in an ethanol bath for three days. For the modified aerogels, the DMF reagent was introduced into the solution at a mole ratio of 1:1 with TEOS in the hydrolysis step and condensation step, resulting in the samples marked as $\mathrm{S}-\mathrm{H}$ and S-C, respectively. For the blank control sample, equal volume of ethanol was added into the contrast sample accordingly. The samples without DMF were marked as S-N.

Characterization: the bulk density of the silica aerogel was determined by measuring the dimensions and mass of the monoliths. The particle size distribution of silica sol was monitored by the dynamic light scattering analysis (DLS, BI-200SM, USA). The microstructure of the aerogels was studied using field-emission scanning electron microscopy (FE-SEM, S-4800, Japan). The chemical composition of silica aerogels was characterized with Fourier transform-infrared spectroscopy (FTIR, Perkin-Elmer, USA). Surface area, pore volume, and pore size distribution (PSD) were measured by $\mathrm{N}_{2}$ adsorptiondesorption method (Autosorb-iQ, USA). The pore volume $\left(V_{\mathrm{N} 2}\right)$ is chosen the single point desorption pore volume of pores size smaller than $100 \mathrm{~nm}$ at $\mathrm{P} / \mathrm{P}_{0}=0.98$. Total pore volume $\left(V_{\mathrm{t}}\right)$ was calculated according to the equation:

$V_{\mathrm{t}}=1 / \rho_{\mathrm{b}}-1 / \rho_{\mathrm{s}}$,

where $\rho_{\mathrm{b}}$ is the bulk density, $\rho_{\mathrm{s}}$ is the skeletal density of silica aerogel which is $2200 \mathrm{~kg} / \mathrm{m}^{3}$.

\section{RESULTS AND DISCUSSION}

Fig. 1 shows the steric shielding effect of DMF on the silica clusters (Fig. $1 \mathrm{~b}$ ) and the DLS analysis results for the silica sol particles size in the sol-gel process after the base addition (20th min) (Fig. $1 \mathrm{c}$ ).

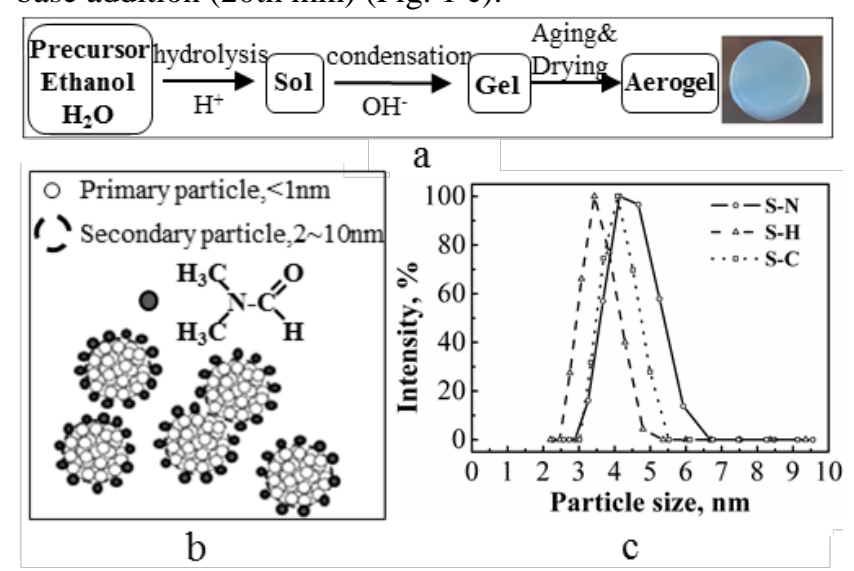

Fig. 1. $a$ - flow chart of the aerogel; $b$ - the shielding effect of DMF on clusters; c-DLS analysis of the sol particles

It can be seen that the distribution of secondary sol particles $(2 \sim 10 \mathrm{~nm})$ of $\mathrm{S}-\mathrm{H}$ and $\mathrm{S}-\mathrm{C}$ are narrower than that of $\mathrm{S}-\mathrm{N}$, indicating that the addition of DMF reagent inhibits the aggregation of primary colloidal particles. This is attributed to the steric shielding around the colloid particles, which is provided by DMF on the surface of particles through hydrogen bond [20]. Meanwhile, the S-H has smaller particle size while compared with S-C. DMF can decrease the hydrolysis rate by attaching with $\mathrm{H}_{2} \mathrm{O}$ molecules. Thus, the growth of silica sol particles in $\mathrm{S}-\mathrm{H}$ will be limited due to the lower hydrolysis degree.

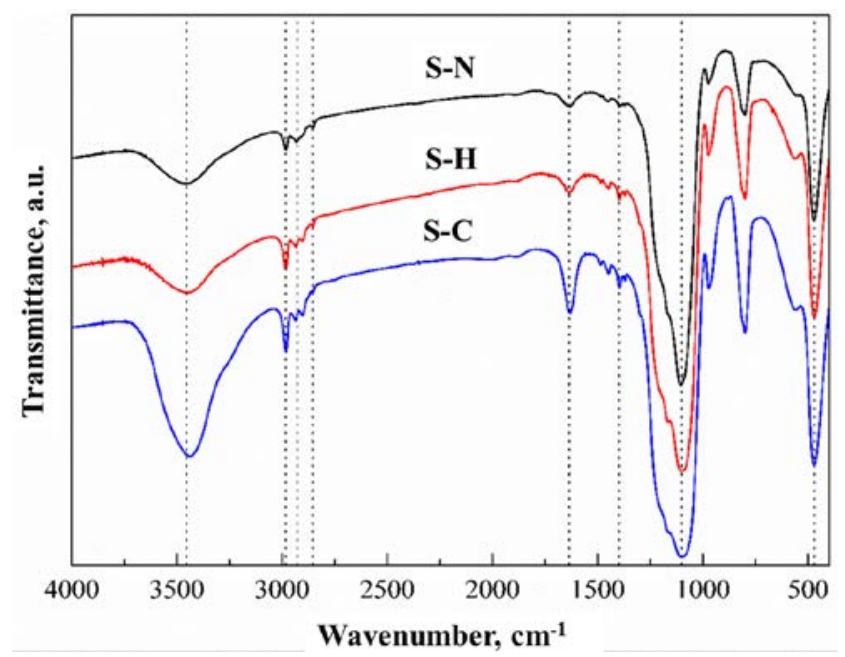

Fig. 2. FTIR spectra of the samples

The FTIR spectra of S-N, S-H and S-C in Fig. 2 exhibit the characteristic absorption peaks located at the same wavenumbers. A broad band located at $3454 \mathrm{~cm}^{-1}$ and a peak at $1634 \mathrm{~cm}^{-1}$ belong to the stretching vibrations of surface $-\mathrm{OH}$ groups and the bending vibration of adsorbed $\mathrm{H}_{2} \mathrm{O}$, respectively. The peaks at $2983 \mathrm{~cm}^{-1}$, $2926 \mathrm{~cm}^{-1}, 2853 \mathrm{~cm}^{-1}$, and $1400 \mathrm{~cm}^{-1}$ correspond to the C$\mathrm{H}$ bonds, indicating the existence of some residual carbonaceous organic groups in the aerogels after the supercritical drying. Besides, the intense bands near $1100 \mathrm{~cm}^{-1}$ and $470 \mathrm{~cm}^{-1}$ are assigned to the Si-O-Si bonds.

SEM images in Fig. 3 exhibit the three-dimensional nanoporous structures of the obtained aerogel samples. When compared with the S-N (Fig. 3 a), the S-H and S-C exhibit larger pore size and smaller particle size (Fig. $3 \mathrm{~b}, \mathrm{c}$ ). This suggests that the aggregation of the colloidal particles have been restrained by DMF effectively. Furthermore, the S-C has more uniform pore structure than sample $\mathrm{S}-\mathrm{H}$. As mentioned above, the DMF can decrease the hydrolysis rate. The wet gel of the $\mathrm{S}-\mathrm{H}$ will hydrolyse and polymerize furtherly in the aging or supercritical drying step, resulting in the heterogeneity of the pore structure in the S-H. Correspondingly, the DMF added in the condensation step has limited effect on hydrolysis process. As a result, the PSD of S-C is more uniform.

$\mathrm{N}_{2}$ adsorption-desorption isotherms of silica aerogels are shown in Fig. 4 a. These isotherms represent a type IV nature according to the IUPAC classification. The hysteresis loops at the high pressure are attributed to the capillary condensation occurring in mesopores. All the isotherms have sharp increase in the relative pressure region of $P / P_{0}=0.95-1.0$, which indicates the liquid condensation related to the presence of macropores [3]. The $\mathrm{N}_{2}$ adsorption values of $\mathrm{S}-\mathrm{H}$ and $\mathrm{S}-\mathrm{C}$ aerogels are much higher than the $\mathrm{S}-\mathrm{N}$ sample. In fact, the nanopore volumes of the $\mathrm{S}-\mathrm{H}$ and $\mathrm{S}-\mathrm{C}$ are as high as $6.0 \mathrm{~cm}^{3} / \mathrm{g}$ and $5.7 \mathrm{~cm}^{3} / \mathrm{g}$ (Table 1), while it is much smaller in the $\mathrm{S}-\mathrm{N}$ aerogel $\left(4.6 \mathrm{~cm}^{3} / \mathrm{g}\right)$. The results illustrate that there are much more nanopores preserved in the aerogel that were obtained with the addition of DMF. 


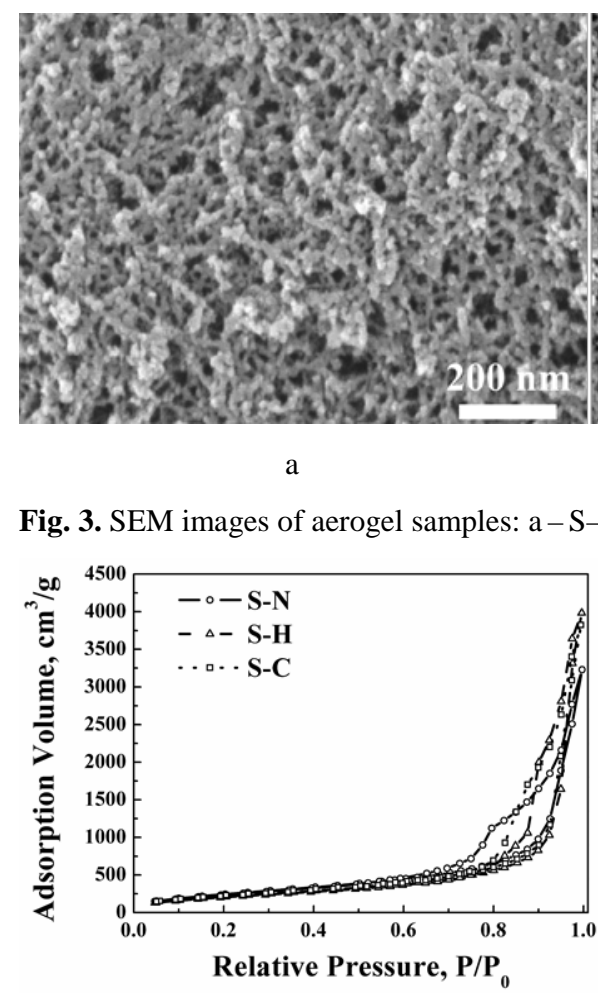

a

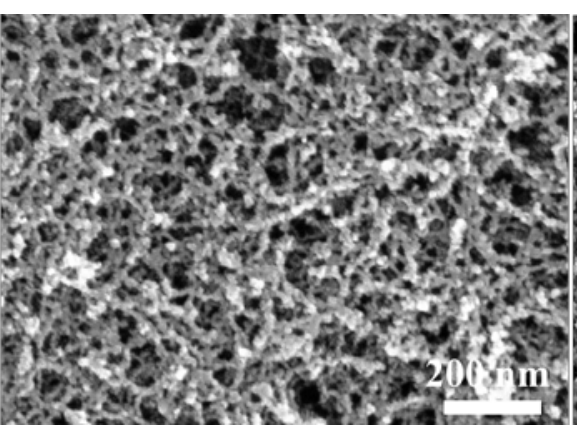

b

C

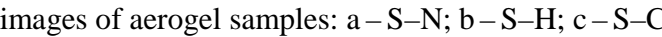

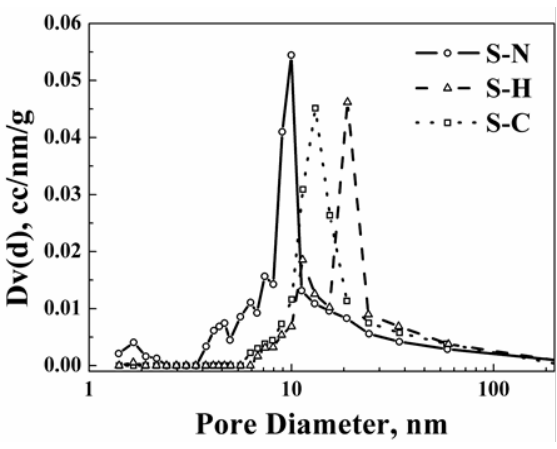

b

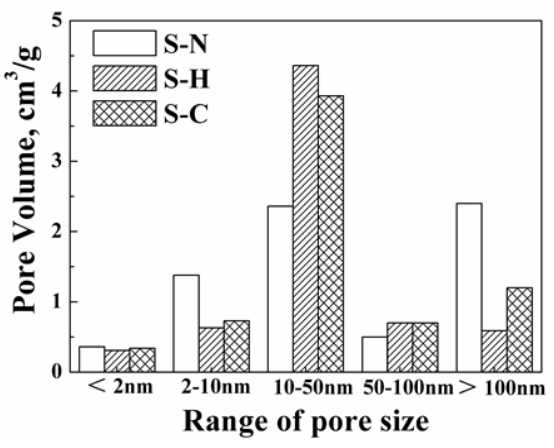

C

Fig. 4. $\mathrm{a}-\mathrm{N}_{2}$ adsorption-desorption isotherms; $\mathrm{b}$-the pore size distribution; $\mathrm{c}$-the pore volume of the samples corresponding to different range of pore size

Table 1. Structural properties of all the silica aerogels

\begin{tabular}{|c|c|c|c|c|}
\hline Samples & $\begin{array}{c}\text { Density, } \\
\mathrm{g} / \mathrm{cm}^{3}\end{array}$ & $\begin{array}{c}\text { Surface area, } \\
\mathrm{m}^{2} / \mathrm{g}\end{array}$ & $\mathrm{V}_{\mathrm{t}}$ & $\mathrm{V}_{\mathrm{N} 2}$ \\
\hline $\mathrm{S}-\mathrm{N}$ & 0.134 & 950 & 7.0 & 4.6 \\
\hline $\mathrm{S}-\mathrm{H}$ & 0.142 & 813 & 6.6 & 6.0 \\
\hline $\mathrm{S}-\mathrm{C}$ & 0.136 & 874 & 6.9 & 5.7 \\
\hline
\end{tabular}

It has been found that the specific surface area values of S-N, S-H and S-C are $950 \mathrm{~m}^{2} / \mathrm{g}, 813 \mathrm{~m}^{2} / \mathrm{g}$ and $874 \mathrm{~m}^{2} / \mathrm{g}$. This is resulted from the acceleration of polymerization reaction after the addition of DMF, making the primary particles become greater [19].

The PSD curves of the aerogels presented in Fig. $4 \mathrm{~b}$ reveal that the $\mathrm{S}-\mathrm{H}$ and $\mathrm{S}-\mathrm{C}$ have larger pore size compared to the S-N. Moreover, the pore size of the S-N are concentrated at $10 \mathrm{~nm}$, while they increase to $19 \mathrm{~nm}$ and $13 \mathrm{~nm}$ for $\mathrm{S}-\mathrm{H}$ and $\mathrm{S}-\mathrm{N}$, respectively. This indicates that the introduction of DMF can enlarge the pore size. Larger pore sizes for the nanopores will provide larger volume. Meanwhile, the S-C shows more uniform PSD compared with the bimodal distribution of the $\mathrm{S}-\mathrm{H}$. The pore volume corresponding to different pore size range is presented in Fig. 4 c. The larger pore volume of the S-N for the pores of $2-10 \mathrm{~nm}$ results from the aggregation of the primarily sol particles. It is obviously to find that there are so many pores larger than $100 \mathrm{~nm}$ in $\mathrm{S}-\mathrm{N}$, while most of pores in $\mathrm{S}-\mathrm{H}$ and $\mathrm{S}-\mathrm{C}$ are located in the range of $10-50 \mathrm{~nm}$. The decrease of pores larger than $100 \mathrm{~nm}$ in $\mathrm{S}-\mathrm{H}$ and $\mathrm{S}-\mathrm{C}$ states that the introduction of DMF have controlled the aggregation of colloidal particles remarkably.

\section{CONCLUSIONS}

Silica aerogels with nanopores volume as high as $6.0 \mathrm{~cm}^{3} / \mathrm{g}$ are synthesized. With the addition of DMF in the condensation period, the content of pores larger than $100 \mathrm{~nm}$ decreases remarkably, owing to the shielding layers of DMF around particles inhibiting the aggregation of the sol particles effectively. Besides, the silica aerogels with more uniform pore size distribution have been obtained with the introduction of DMF in condensation step rather than the hydrolysis step. This study provides an effective approach for the preparation of silica aerogels with high nanopore volume and uniform pore structure. Next, our researches will focus on the effects of high nanopore volume and uniform pore structure on aerogel performance, especially the thermal insulation performance.

\section{REFERENCES}

1. Kocon, L., Despetis, F., Phalippou, J. Ultralow Density Silica Aerogels by Alcohol Supercritical Drying Journal of Non-Crystalline Solids 225 1998: pp. 96-100. https://doi.org/10.1016/S0022-3093(98)00322-6

2. Sarawade, P., Kim, J., Kim, H., Kim, H. High Specific Surface Area TEOS-based Aerogels with Large Pore Volume Prepared at an Ambient Pressure Applied Surface Science 254 (2) 2007: pp. 574-579. https://doi.org/10.1016/j.apsusc.2007.06.063

3. Zhao, S., Zhang, Z., $\quad$ Gilles, S., $\quad$ Rudder, W., Raymond, V.R., Philippe, T., Matthias, M.K. Multiscale 
Assembly of Superinsulating Silica Aerogels Within Silylated Nanocellulosic Scaffolds: Improved Mechanical Properties Promoted by Nanoscale Chemical Compatibilization Advanced Functional Materials 25 (15) 2015: pp. 2326-2334.

https://doi.org/10.1002/adfm.201404368

4. Ellann, C., Leon, G. Thermal Properties of Silica Aerogel Formula Journal of Heat Transfer 137 (8) 2015: pp. $1-8$. https://doi.org/10.1115/1.4028901

5. Xia, T., Yang, H., Li, J., Sun, C., Lei, C., Hu, Z., Zhang, Y. Tailoring Structure and Properties of Silica Aerogels by Varying the Content of the Tetramethoxysilane Added in Batches Microporous and Mesoporous Materials 280 2019: pp. $20-25$

https://doi.org/10.1016/j.micromeso.2019.01.038

6. Wei, G., Zhang, Y., Xu, C., Du, X., Yang, Y. A Thermal Conductivity Study of Double-Pore Distributed Powdered Silica Aerogels International Journal of Heat and Mass Transfer 108 2017: pp. 1297-1304. https://doi.org/10.1016/j.ijheatmasstransfer.2016.12.062

7. Feng, J., Le, D., Nguyen, S., Nien, V., Jewell, D., Duong, H. Silica-cellulose Hybrid Aerogels for Thermal and Acoustic Insulation Applications Colloids and Surfaces A: Physicochemical and Engineering Aspects 506 2016: pp. $298-305$. https://doi.org/10.1016/j.colsurfa.2016.06.052

8. Cai, J., Lucas, S., Wang, L., Cao, Y. Insulation Properties of the Monolithic and Flexible Aerogels Prepared at Ambient Pressure Advanced Materials Research 391-392 2012: pp. $116-120$. https://doi.org/10.4028/www.scientific.net/AMR.391392.116

9. Pierre, A., Pajonk, G. Chemistry of Aerogels and Their Applications 2002: pp. $4243-4265$.

https://doi.org/10.1021/cr0101306

10. Zu, G., Kanamori, K., $\quad$ Nakanishi, K., Huang, J. Superhydrophobic Ultraflexible Triple-Network Graphene/Polyorganosiloxane Aerogels for a HighPerformance Multifuncational Temperature/Strain/Pressure Sensing Array Chemistry of Materials 31 2019: pp. 6276-6285.

https://doi.org/10.1021/acs.chemmater.9b02437

11. Lin, D., Yuen, P., $\quad$ Liu, Y., $\quad$ Liu, W., $\quad$ Liu, N., Dauskardt, R., Cui, Y. A Silica-Aerogel-Reinforced Composite Polymer Electrolyte with High Ionic Conductivity and High Modulus Advanced Materials 30 (32) 2018, 1802661 pp. 1-8. https://doi.org/10.1002/adma.201802661

12. Zu, G., Shen, J., Wang, W., Zou, L., Lian, Y., Zhang, Z. Silica-Titania Composite Aerogel Photocatalysts by Chemical Liquid Deposition of Titania onto Nanoporous Silica Scaffolds ACS Applied Materials and Interfaces 7 2015: pp. 5400-5409. https://doi.org/10.1021/am5089132
13. Huber, L., Zhao, S., Malfait, W., Vares, S., Koebel, M. Fast and Minimal-Solvent Production of Superinsulating Silica Aerogel Granulate Angewandte Chemie International Edition 56 2017: pp. 1-5.

https://doi.org/10.1002/anie.201700836

14. Zu, G., Shimizu, T., Kanamori, K., Zhu, Y., Maeno, A., Kaji, H., Shen, J., Nakanishi, K. Transparent, Superflexible Doubly Cross-Linked Polyvinylpolymethylsiloxane Aerogel Superinsulators via Ambient Pressure Drying ACS Nano 12 (1) 2018: pp. 521-532. https://doi.org/10.1021/acsnano.7b07117

15. Deng, Z., Wang, J., Wei, J., Shen, J., Zhou, B., Chen, L. Physical Properties of Silica Aerogels Prepared with Polyethoxydisiloxanes Journal of Sol-Gel Science and Technology 19 2000: pp. 677-680. https://doi.org/10.1023/A:1008754504788

16. Liu, G., Zhou, B., Du, A., Shen, J., Wu, G. Greatly Strengthened Silica Aerogels Via Co-Gelation of Binary Sols with Different Concentrations: A Method to Control the Microstructure of The Colloids Colloids and Surfaces A: Physicochem Eng Aspects 436 2013: pp. 763-774. https://doi.org/10.1016/j.colsurfa.2013.08.003

17. Haranath, D., Rao, A., Wagh, P. Influence of DCCAs on Optical Transmittance and Porosity Properties of TMOS Silica Aerogels Journal of Porous Materials 6 1999: pp. 55-62. https://doi.org//10.1023/A:1009649403740

18. He, S., Huang, D., Bi, H., Li, Z., Yang, H., Cheng, $X$. Synthesis and Characterization of Silica Aerogels Dried Under Ambient Pressure Bed on Water Glass Journal of Non-Crystalline Solids 410 2015: pp. 58-64. https://doi.org/10.1016/j.jnoncrysol.2014.12.011

19. Rao, A., Sakhare, H., Tamhankar, A., Shinde, M., Gadave, D., Wagh, P. Influence of $\mathrm{N}$, Ndimethylformamide Additive on the Physical Properties of Citric Acid Catalyzed TEOS Silica Aerogels Materials Chemistry and Physics 60 1999: pp. $268-273$. https://doi.org/10.1016/S0254-0584(99)00089-9

20. Cai, L., Shan, G. Elastic Silica Aerogel Using Methyltrimethoxysilane Precusor Via Ambient Pressure Drying Journal of Porous Materials 22 (6) 2015: pp. $1455-1463$. https://doi.org/10.1007/s10934-015-0026-6

21. Chiappim, W.Jr., Awano, C., Donatti, D., De Vicente, F., Vollet, D. Structure of Hydrophobic Ambient-PressureDried Aerogels Prepared by Sonohydrolysis of Tetraethoxysilane With Additions of N, Ndimethylformamide Langmuir 30 (4) 2014: pp. $1151-1159$. https://doi.org/10.1021/la403798t

22. Vollet, D., De Sousa, W., Donatti, D., Ruiz, A. Mass Fractal Characteristics of Sonogels Prepared from Sonohydrolysis of Tetraethoxysilane with Additions of Dimethylformamide Journal of Non-Crystalline Solids 353 (2) 2007: pp. 143-150. https://doi.org/10.1016/j.jnoncrysol.2006.09.038 